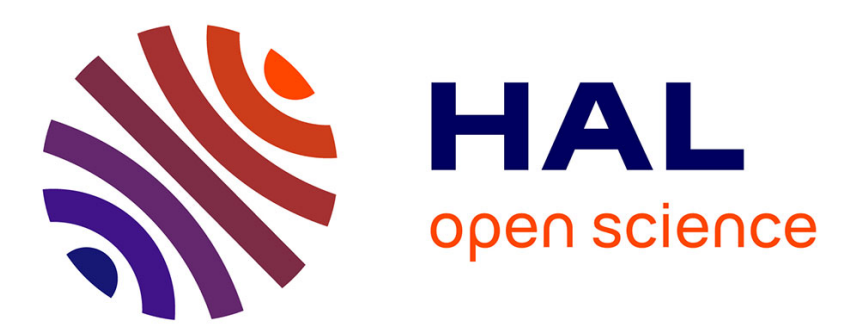

\title{
Leveraging Dynamic Occupancy Grids for 3D Object Detection in Point Clouds
}

David Sierra González, Anshul Paigwar, Özgür Erkent, Jilles Dibangoye, Christian Laugier

\section{- To cite this version:}

David Sierra González, Anshul Paigwar, Özgür Erkent, Jilles Dibangoye, Christian Laugier. Leveraging Dynamic Occupancy Grids for 3D Object Detection in Point Clouds. ICARCV 2020 - 16th IEEE International Conference on Control, Automation, Robotics and Vision, Dec 2020, Shenzhen, China. pp.1-6. hal-03044979

\author{
HAL Id: hal-03044979 \\ https://hal.inria.fr/hal-03044979
}

Submitted on 7 Dec 2020

HAL is a multi-disciplinary open access archive for the deposit and dissemination of scientific research documents, whether they are published or not. The documents may come from teaching and research institutions in France or abroad, or from public or private research centers.
L'archive ouverte pluridisciplinaire HAL, est destinée au dépôt et à la diffusion de documents scientifiques de niveau recherche, publiés ou non, émanant des établissements d'enseignement et de recherche français ou étrangers, des laboratoires publics ou privés. 


\section{Leveraging Dynamic Occupancy Grids for 3D Object Detection in Point Clouds}

David Sierra-González, Anshul Paigwar, Ozgur Erkent, Jilles Dibangoye, and Christian Laugier
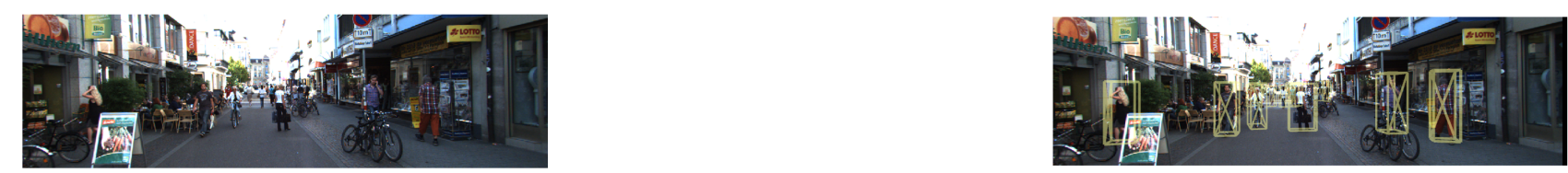

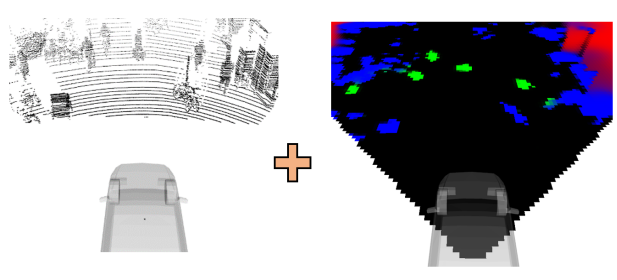

3D point cloud Dynamic Occupancy Grid

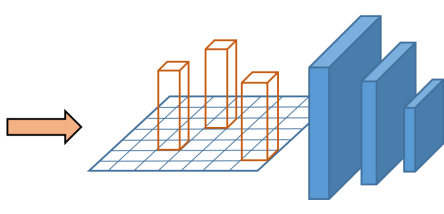

PointPillars

Architecture

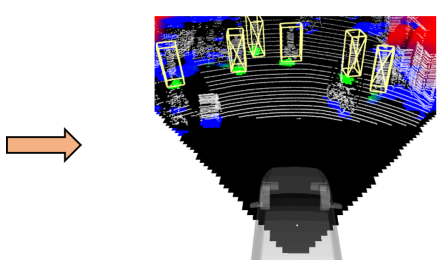

3D multiclass bounding box prediction

Fig. 1: 3D object detection in point clouds leveraging dynamic occupancy grid representations of the environment.

\begin{abstract}
Traditionally, point cloud-based 3D object detectors are trained on annotated, non-sequential samples taken from driving sequences (e.g. the KITTI dataset). However, by doing this, the developed algorithms renounce to exploit any dynamic information from the driving sequences. It is reasonable to think that this information, which is available at test time when deploying the models in the experimental vehicles, could have significant predictive potential for the object detection task. To study the advantages that this kind of information could provide, we construct a dataset of dynamic occupancy grid maps from the raw KITTI dataset and find the correspondence to each of the KITTI 3D object detection dataset samples. By training a Lidar-based state-of-the-art 3D object detector with and without the dynamic information we get insights into the predictive value of the dynamics. Our results show that having access to the environment dynamics improves by $27 \%$ the ability of the detection algorithm to predict the orientation of smaller obstacles such as pedestrians. Furthermore, the 3D and bird's eye view bounding box predictions for pedestrians in challenging cases also see a $7 \%$ improvement. Qualitatively speaking, the dynamics help with the detection of partially occluded and far-away obstacles. We illustrate this fact with numerous qualitative prediction results.
\end{abstract}

\section{INTRODUCTION AND RELATED WORK}

Perceiving the environment in a reliable manner is one of the hardest challenges that needs to be solved before the commercial deployment of fully autonomous vehicles becomes a reality. Amongst other perception tasks, autonomous robots need to detect static and moving objects in the 3D world, under varying weather and lighting conditions. To perform

\footnotetext{
This work was supported by Toyota Motor Europe.

${ }^{1}$ The authors are with Univ. Grenoble-Alpes, Inria, 38000 Grenoble, France - \{david.sierra-gonzalez, anshul.paigwar, ozgur.erkent, jilles.dibangoye, christian.laugier\}@inria.fr
}

this task, different sensing technologies are available, namely, monocular and stereo cameras, Lidar scanners and radar.

The avalanche of novel deep learning techniques developed over the past decade have brought the $2 \mathrm{D}$ object detection task very close to be considered a solved problem [1], [2], [3]. However, in robotics, and more particularly in the domain of autonomous driving, we are more interested in detecting objects in 3D; that is, we aim to find oriented $3 \mathrm{D}$ bounding boxes around the obstacles. Unfortunately, the progress in $3 \mathrm{D}$ obstacle detection methods is lagging behind their $2 \mathrm{D}$ counterparts.

To improve the situation, different sensing modalities are being explored. For example, some approaches aim to detect the 3D obstacles relying exclusively in monocular or stereo images [4]. However, it seems that the characteristics of Lidar sensors are more suitable for this perception task, as evidenced by the ranking of the KITTI 3D object detection benchmark [5]. Lidars can directly provide range information and are unaffected by lighting conditions. Nonetheless, the point cloud data these sensors provide is sparse, and it becomes increasingly so at larger distances.

Different detection approaches building upon point cloud data have been introduced in recent years. Since the point cloud is sparse, a common technique is to voxelize the $3 \mathrm{D}$ space to create a tensor on which to perform convolutional operations and transfer techniques from the 2D detection field. Li proposes to encode each voxel with the occupancy value of the point cloud and then apply 3D convolutions [6]. Other authors opt for a top-view representation of the point cloud, in which the height corresponds to hand-engineered features such as the height map of the points, the point density and the reflectance at the maximum height [7], [8].

Instead of relying on hand-crafted features, other au- 
thors use the PointNet architecture to automatically learn discriminative point cloud features relevant for the object detection task [9], [10], [11]. The VoxelNet architecture voxelizes the $3 \mathrm{D}$ space and encodes the points inside each voxel with features learned with PointNet; this point cloud representation is followed by $3 \mathrm{D}$ convolutions, a backbone network, and a single-stage detection head [10]. The PointPillars architecture follows a similar approach but avoids the expensive 3D convolution by representing the point cloud from the top view, where the height dimension corresponds to the PointNet features.

One of the common characteristics of all the cited approaches is that none of them exploit the sequential nature of the sensor measurements, i.e. with each new sample they forget everything they saw before. One explanation for this is that the KITTI dataset, the gold standard of perception datasets for robotics and autonomous driving, only provides non-sequential samples in the detection benchmarks. If no sequential information is provided, then the dynamics of the environment cannot be leveraged for the detection task.

To the best of our knowledge, the only approach that considers successive point cloud measurements with a detection objective is Fast and Furious [12]. This approach jointly addresses the object detection, tracking, and prediction tasks. Its key idea is to stack together voxelized representations of a given number of successive past point clouds and perform $3 \mathrm{D}$ convolutions over space and time to predict bounding boxes for future frames. The authors claim that this approach leads to improved detection of occluded and far-away obstacles.

Although not directly detecting on point clouds, Hoermann et al. propose to perform bird's eye view (BEV) bounding box predictions using dynamic occupancy grid environment representations of each sample [13]. Their architecture consists of an encoder-decoder with single-stage detection head. Their results show that they can successfully predict $\mathrm{BEV}$ bounding boxes for cars and pedestrians on a dataset collected from a stationary platform. It is unclear whether this approach would scale to datasets with a more diverse collection of traffic scenes.

In this paper, we perform an in-depth study on how leveraging the dynamics of a driving scene can affect the 3D object detection capabilities of a state-of-art detection architecture on the KITTI dataset. We hypothesize, in line with previous work [12], that this information has significant predictive potential for the object detection task. To perform our study, we extend the KITTI 3D object detection dataset with a dynamic occupancy grid for each sample [5].

The rest of the document is organized as follows: Section II describes the proposed experiment and provides basic information about dynamic occupancy grids and about the 3D detection architecture that will be tested; Section III describes the dataset that has been prepared to provide dynamic information of the environment in the KITTI 3D object detection dataset. This section also presents and discusses the different qualitative and quantitative experimental results. Lastly, Section IV concludes.

\section{3D OBJECT DETECTION IN POINT CLOUDS WITH DYNAMIC INFORMATION}

To determine if Lidar-based 3D object detection algorithms can benefit from having access to the dynamic information of a scene, we extend the 3D KITTI object detection dataset with the dynamic occupancy grid representation of the environment for each of the samples. We describe the constructed dataset in subsection III-A. In this section, we provide details about the tracker used to generate the grid representations and about how we can combine these representations with the features that encode the point cloud data.

\section{A. Bayesian occupancy grid filtering}

Bayesian occupancy grid filtering is a technique for situational awareness in which the environment is represented as a grid of cells and the occupancy state of each cell is maintained over time [14], [15]. By processing measurements over time, this technique can estimate the velocity of each cell in the grid, modeling thus the dynamic environment.

To produce the dynamic occupancy grids (also known as dynamic occupancy grid maps, or DOGMas) for the KITTI dataset we use the Conditional Monte Carlo Dense Occupancy Tracker (CMCDOT) [16]. This approach uses the Lidar measurements to maintain two distinct discrete probability distributions for each cell, one over its occupancy state and the other over the velocity of the cell's occupancy. The possible occupancy states for a cell are: "occupied by a static object", "occupied by a dynamic object", "empty" and "unknown". Figs. 1-3 show visualizations of some of the DOGMas of the KITTI validation split. The color scheme is the following: the red channel is for the unknown probability, green is for the dynamic occupancy probability, and blue is for the static occupancy probability. Thus, cells colored in black are estimated to be empty. The cells outside the FOV of the camera are displayed in white.

\section{B. Leveraging DOGMAs for $3 D$ object detection}

Fig. 2 shows an overview of the proposed architecture used to study the impact of dynamic data in the 3D object detection performance. After receiving a Lidar point cloud measurement, we can obtain the DOGMa environment representation with the occupancy tracker. Using the FeatureNet component of the PointPillars architecture (in essence, a simplified version of PointNet [9]), we can also produce a tensor known as pseudo-image that characterizes the distribution of Lidar points above each cell [11]. We then concatenate both environment representations and pass them through the backbone and SSD detection head to produce the 3D bounding boxes and the class predictions [2].

The DOGMa calculation on GPU is about $50 \mathrm{~ms}$ for the selected grid size and tracker parameters. However, this calculation can be executed in parallel with the point cloud preprocessing and the pseudo-image generation, which take up roughly $60 \%$ of the PointPillars total runtime. In any case, the DOGMa computation time could be significantly reduced by trading-off grid accuracy. 


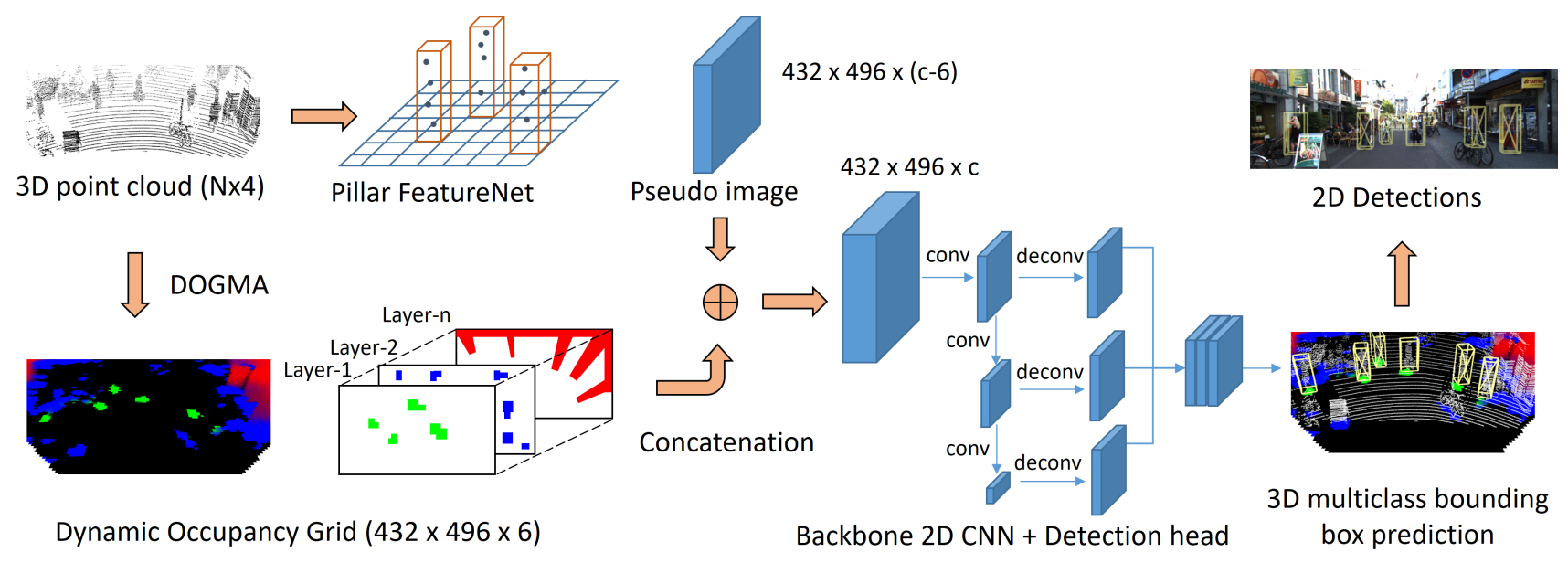

Fig. 2: Overview of the proposed experimental architecture. Each point cloud from the KITTI 3D object detection dataset has an associated DOGMa obtained from the KITTI raw dataset. Using PointPillar's point cloud feature extractor, a tensor that matches the size of the DOGMa is obtained and concatenated with the DOGMa features. The resulting tensor is passed through the backbone network and the SSD detector head produces the 3D bounding box predictions

\section{EXPERIMENTAL EVALUATION}

In this section, we first detail the characteristics of the constructed DOGMa dataset and the settings selected for the PointPillars architecture and for the experimental evaluation. Next, we present and discuss the quantitative results obtained. Finally, we present several qualitative results to help illustrate the main points of the paper.

\section{A. Dataset construction}

The KITTI 3D object detection dataset consists of 7481 training images and 7518 test images, as well as their corresponding point clouds, with a total of 80.256 labeled objects. The training set is typically split into 3712 training samples and 3769 validation samples [17], [11]. To find the DOGMas corresponding to each of the training and validation samples, we run the CMCDOT dynamic occupancy grid filter on all the driving sequences of the KITTI raw dataset. The mapping from each training and validation sample to the corresponding driving sequence sample is provided. For the test samples the mapping is not provided, so we will not be able to benchmark our approach on the test set.

The selected DOGMa size was $(432,496)$ cells, with a resolution of $0.16 \mathrm{~m}$. This corresponds to a distance of $69.12 \mathrm{~m}$ along the $x$ axis (front of the vehicle) and $39.68 \mathrm{~m}$ to each side in the $y$ axis. Each cell has six associated floating point values, namely, the probabilities of the four possible states of the cell (occupied static or dynamic, free, unknown), and the estimated velocities for the cell's occupancy along the $x$ and $y$ axes. The KITTI dataset provides no labels for objects outside the field of view (FOV) of the camera. All cells lying outside the FOV have their values set to 0 .

As the CMCDOT filtering requires a few steps before convergence, the DOGMas of some of the training and validation samples that correspond to the beginning of the driving sequences could not be obtained. Also, the odometry of one of the driving sequences of the raw dataset appeared to be corrupted, so the DOGMa could not be calculated for any of the samples taken from that sequence. All in all, the final number of training and validation samples is reduced to 3705 and 3570, respectively. The complete DOGMa dataset will be released upon publication ${ }^{1}$.

\section{B. $3 D$ detection architecture and experimental settings}

The settings selected for the PointPillars architecture correspond in general to the default values of the PyTorch opensource code released by the authors of the paper ${ }^{2}$. The cell resolution is also set to $0.16 \mathrm{~m}$ and the point cloud limits are selected so that the size of the pseudo-image matches the size of the generated DOGMas. Some of the more relevant parameters are the maximum number of voxels, which is set to 12.000 ; the maximum number of points per voxel, set to 100; and the number of features automatically learned per cell ( $c-6$ in Fig. 2), which we set to 64 .

A model is trained for the car class and another for the pedestrian/cyclist classes. We train each model for 160 epochs. The evaluation is performed on the validation set. Following previous work, we perform object and global augmentation in each sample during training [18], [10], [11]. Object augmentation refers to inserting artificial instances of the target object class in each sample. The artificial instances are sampled from a database constructed using the original dataset. In our case, each artificial instance consists of the object's point cloud as well as the DOGMa cells below the points. Note that we do not insert the trail of dynamic occupancy that lags behind objects moving at medium to large speeds.

\section{Quantitative results}

To evaluate the impact of the DOGMa features in the obstacle detection task, we consider three different metrics. First, we look at the Average Precision (AP) of the 3D

\footnotetext{
${ }^{1}$ https://github.com/d451gon/kitti_dogma_dataset

${ }^{2}$ https://github.com/nutonomy/second.pytorch
} 


\begin{tabular}{|c|c|c|c|c|c|c|c|c|c|c|c|c|}
\hline \multicolumn{2}{|c|}{ Features } & \multicolumn{2}{c|}{ mAP } & \multicolumn{3}{c|}{ Car } & \multicolumn{3}{c|}{ Pedestrian } & \multicolumn{3}{c|}{ Cyclist } \\
\hline Pointnet & Grid & Mod. & Hard & Easy & Mod. & Hard & Easy & Mod. & Hard & Easy & Mod. & Hard \\
\hline $\boldsymbol{X}$ & $\checkmark$ & 13.44 & 13.44 & 20.74 & 16.45 & 15.48 & 17.75 & 15.46 & 14.99 & 11.57 & 08.41 & 08.21 \\
\hline$\checkmark$ & $\boldsymbol{X}$ & $\mathbf{6 4 . 9 2}$ & $\mathbf{5 9 . 3 3}$ & $\mathbf{8 4 . 0 6}$ & $\mathbf{7 5 . 1 3}$ & $\mathbf{6 9 . 4 3}$ & $\mathbf{6 2 . 5 7}$ & 57.52 & 51.17 & $\mathbf{8 1 . 9 6}$ & $\mathbf{6 2 . 1 1}$ & $\mathbf{5 7 . 3 9}$ \\
\hline$\checkmark$ & $\checkmark$ & 58.50 & 56.56 & 80.45 & 67.57 & 66.30 & 61.64 & $\mathbf{5 7 . 8 9}$ & $\mathbf{5 5 . 7 8}$ & 69.01 & 50.06 & 47.60 \\
\hline
\end{tabular}

TABLE I: Average precision results on the validation split of the KITTI 3D detection dataset

\begin{tabular}{|c|c|c|c|c|c|c|c|c|c|c|c|c|}
\hline \multicolumn{2}{|c|}{ Features } & \multicolumn{2}{|c|}{ mAP } & \multicolumn{3}{c|}{ Car } & \multicolumn{3}{c|}{ Pedestrian } & \multicolumn{3}{c|}{ Cyclist } \\
\hline Pointnet & Grid & Mod. & Hard & Easy & Mod. & Hard & Easy & Mod. & Hard & Easy & Mod. & Hard \\
\hline $\boldsymbol{X}$ & $\checkmark$ & 29.75 & 28.11 & 56.32 & 43.26 & 39.93 & 26.33 & 23.43 & 22.60 & 30.35 & 22.56 & 21.80 \\
\hline$\checkmark$ & $\boldsymbol{X}$ & $\mathbf{7 2 . 6 3}$ & $\mathbf{6 9 . 0 1}$ & $\mathbf{8 9 . 9 9}$ & $\mathbf{8 7 . 1 3}$ & $\mathbf{8 5 . 1 5}$ & 70.54 & 65.70 & 60.18 & $\mathbf{8 5 . 0 7}$ & $\mathbf{6 5 . 0 6}$ & $\mathbf{6 1 . 7 2}$ \\
\hline$\checkmark$ & $\checkmark$ & 68.00 & 66.44 & 89.54 & 81.91 & 81.83 & $\mathbf{7 2 . 6 4}$ & $\mathbf{6 7 . 5 9}$ & $\mathbf{6 5 . 9 2}$ & 72.55 & 54.51 & 51.58 \\
\hline
\end{tabular}

TABLE II: Average precision results on the validation split of the KITTI Bird's Eye View detection dataset

\begin{tabular}{|c|c|c|c|c|c|c|c|c|c|c|c|c|}
\hline \multicolumn{2}{|c|}{ Features } & \multicolumn{2}{|c|}{ mAP } & \multicolumn{3}{c|}{ Car } & \multicolumn{3}{c|}{ Pedestrian } & \multicolumn{3}{c|}{ Cyclist } \\
\hline Pointnet & Grid & Mod. & Hard & Easy & Mod. & Hard & Easy & Mod. & Hard & Easy & Mod. & Hard \\
\hline $\boldsymbol{X}$ & $\checkmark$ & 21.47 & 21.27 & 40.87 & 31.08 & 29.69 & 14.04 & 12.47 & 13.01 & 28.26 & 20.87 & 21.13 \\
\hline$\checkmark$ & $\boldsymbol{X}$ & $\mathbf{6 5 . 2 9}$ & $\mathbf{6 3 . 3 3}$ & $\mathbf{9 0 . 5 8}$ & $\mathbf{8 8 . 2 5}$ & $\mathbf{8 6 . 2 9}$ & 42.51 & 41.98 & 40.47 & $\mathbf{8 4 . 6 7}$ & $\mathbf{6 5 . 6 5}$ & $\mathbf{6 3 . 2 5}$ \\
\hline$\checkmark$ & $\checkmark$ & 64.70 & 62.12 & 90.01 & 85.64 & 81.86 & $\mathbf{5 5 . 7 1}$ & $\mathbf{5 3 . 1 5}$ & $\mathbf{5 0 . 5 5}$ & 75.19 & 55.32 & 53.96 \\
\hline
\end{tabular}

TABLE III: Average orientation similarity results on the validation split of the KITTI 3D detection dataset

bounding box prediction task; these results are shown in Tab. I. The performance evaluation is separated by classes and also by difficulty. The difficulty depends on different aspects of the obstacle, such as its distance to the ego-vehicle, its level of occlusion, and how truncated it is out of the FOV of the camera.

In the top row of Tab. I we show the results obtained when the only features passed to the backbone network are those of the DOGMa. As we can see, it is not possible to detect 3D objects solely from occupancy grid information.

The second and third rows in Tab. I show the results obtained by the model with point cloud features but without DOGMa features, and the model with both point cloud and DOGMa features, respectively. We can observe that the DOGMa features provide no advantage for the 3D bounding box prediction task in the car and cyclist classes.

In contrast, these features provide an edge for the pedestrian class in the moderate and, especially, in the hard categories. Roughly $25 \%$ of the pedestrians in the KITTI dataset are occluded to some degree, while the number climbs to roughly $40 \%$ for cars [5]. Inspecting the dataset we see that the difference between both classes is that most pedestrians appear to be moving, while there is a large number of cars that are static. This leads us to hypothesize that having access to the DOGMa features, and in particular to the dynamicrelated ones, helps in the detection of partially occluded, moving obstacles. This makes sense, as a partially occluded pedestrian should be able to be detected from a reasonablysized dynamic occupancy and a number of Lidar hits consistent with the average class size. This intuition carries over to obstacles located far-away from the ego-vehicle. It is not clear why the performance improvement does not apply also to cyclists. The number of cyclists in the dataset is about one fourth the number of pedestrians, but there is no information about their occlusion statistics. A possibility is that the object augmentation procedure, in which the dynamic occupancy trail behind fast moving objects was not inserted, might have harmed the cyclist model performance.

Table II provides the AP results for the BEV detection task. The results are consistent with the 3D metrics discussed above.

Finally, Tab. III presents the Average Orientation Similarity metric, which measures how well the heading of the predictions matches the ground truth. Having access to the dynamics of a moving obstacle should facilitate the task of predicting its orientation. In our case, we observe a significantly superior performance for the pedestrian class, which is typically the class whose orientation is harder to predict based only on point cloud data. However, the performance drops in the hard category of the car class (probably connected with noise present in the DOGMa in distant regions) and in all categories of the cyclist class, which did not converge (jointly with the pedestrian class) to a good model.

\section{Qualitative results}

Figure 3 shows several instances of the predictions obtained in the validation set. In Figs. 3a-3i we show the predictions for the model with point cloud and DOGMa features (yellow) and the one without DOGMa features (purple). More particularly, Figs. 3a-3d show scenes in which at least one of the obstacles is occluded. In Fig. 3a, a pedestrian is largely occluded behind a plant, receiving Lidar hits only in the head and upper torso. However, this number of Lidar hits is enough to track the dynamic occupancy by the CMCDOT tracker, leading to a positive detection with the right orientation. The model without DOGMa features fails to detect this pedestrian. Similar situations occur in Figs. 3b through $3 \mathrm{~d}$.

The difficulty of predicting the orientation of an obstacle from point cloud data without any access to dynamics is highlighted in Figs. 3e-3g. Of particular interest is the case of Fig. 3f; predicting the orientation of a pedestrian facing away from the ego-vehicle solely from point cloud data does 


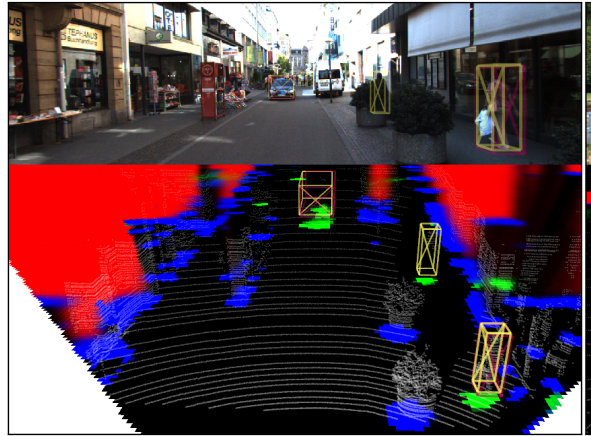

(a) Occluded pedestrian behind the plant.

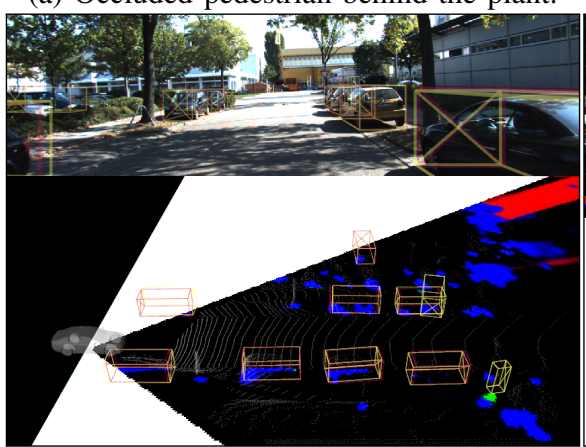

(d) Occluded pedestrian behind vehicles.

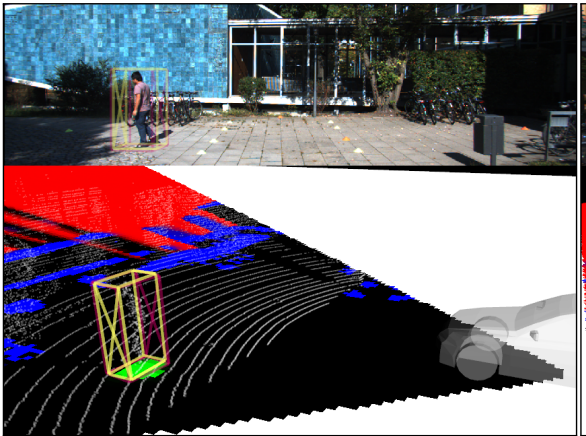

(g) Pose prediction of crossing pedestrian.

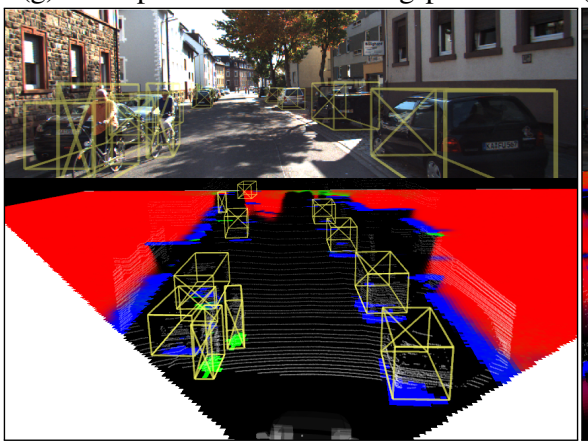

(j) Exemplary prediction on a residential road.

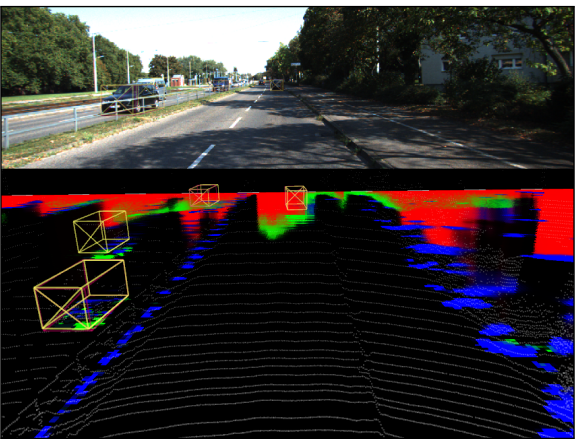

(b) Occluded vehicle by another vehicle.

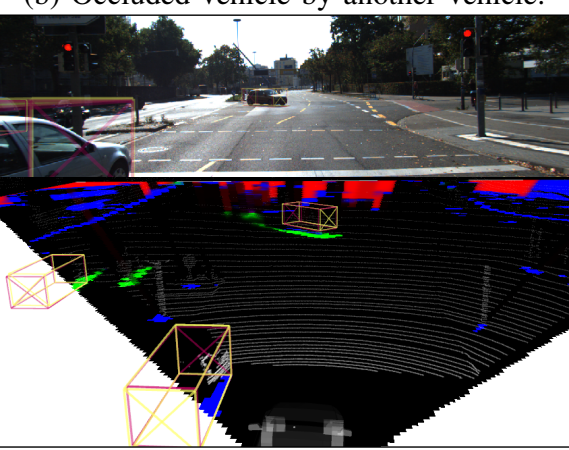

(e) Pose prediction of crossing vehicle.

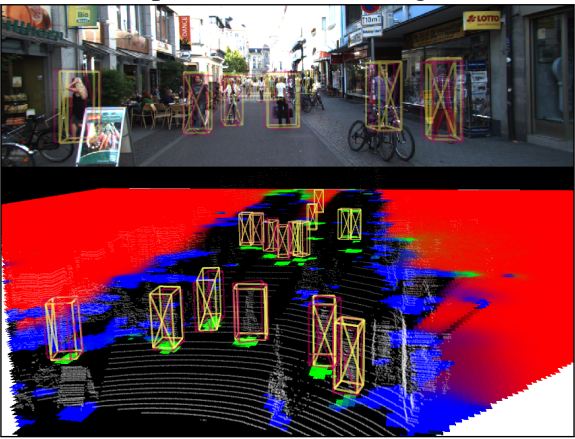

(h) Detecting far-away pedestrians in a crowd.
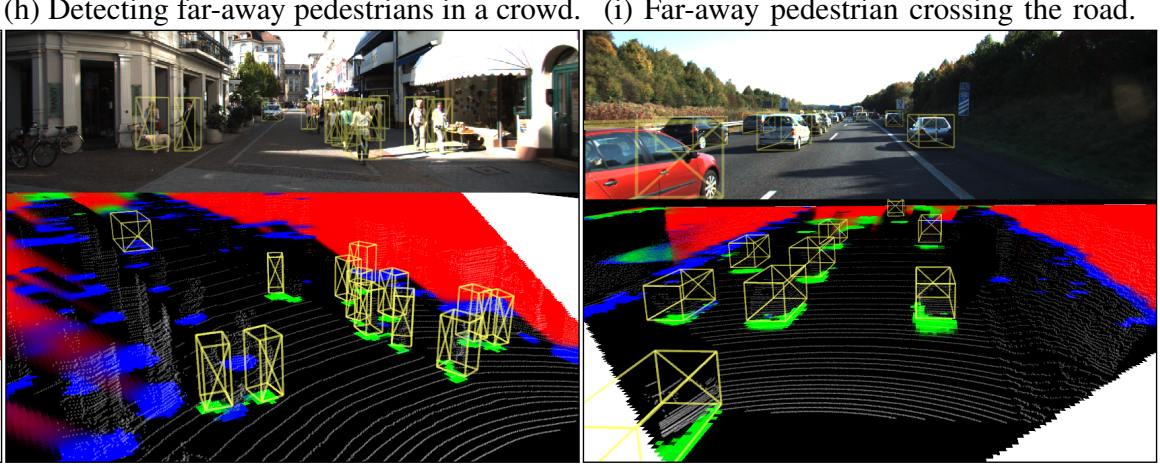

(f) Pose prediction of pedestrian facing away.

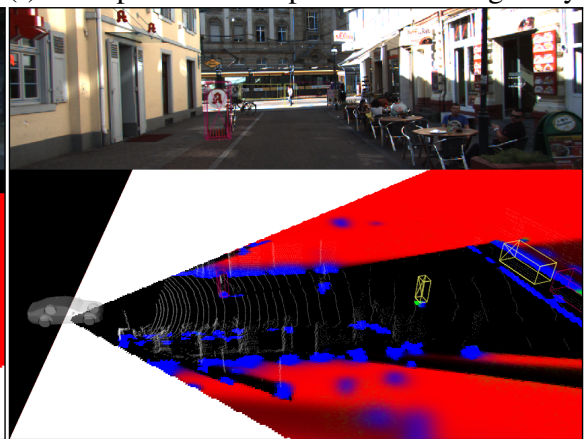

(i) Far-away pedestrian crossing the road.

Fig. 3: Qualitative results obtained in different scenes from the validation split of the KITTI 3D object detection dataset. The ground colors represent the state of each cell of the DOGMa (blue: occupied static, green: occupied dynamic, black: free, red: unknown). Figures (a-i) show the bounding box predictions of the models with DOGMa features (yellow) and without (purple). Figures (j-1) show exemplary predictions of the model with DOGMa features. The first 9 figures aim to show the advantage given by the extra features in the detection of: occluded obstacles (a-d), the orientation of moving obstacles (e-g) and far-away obstacles (h-i).

not seem like an easy task, especially as the distance with the obstacle increases.
Figures $3 \mathrm{~h}$ and $3 \mathrm{i}$ illustrate detections of far-away pedestrians, which are facilitated by the same reasons discussed in 
the case of occluded obstacles. Finally, Figs. 3j-31 present some further exemplary predictions of the model with DOGMa features in different road-network settings.

A video showing the predictions produced by the proposed model in different sequences of the KITTI raw dataset is available at: https://youtu.be/hbegpRuvuUo.

\section{CONCLUSIONS}

In this paper, we studied the impact of combining a dynamic representation of the environment along with point cloud feature descriptors as inputs to a 3D object detection architecture. To achieve this, we created a dataset of DOGMas for the KITTI 3D object detection dataset by running an occupancy tracker on the KITTI raw dataset and using the provided correspondence between both datasets.

With the KITTI dataset enhanced by the dynamic representation of the environment, we trained the PointPillars 3D object detection architecture with different combinations of input features. In light of the detection results, we reached several conclusions. Firstly, using only the occupancy grid features as the input to the detector is not sufficient to produce acceptable 3D or even BEV bounding box predictions for a dataset with diverse traffic scenarios such as KITTI.

Secondly, including dynamic information as input data leads to a significant improvement in the orientation predictions for small obstacles with less points such as pedestrians.

Finally, the 3D and BEV AP detection metrics for the pedestrian class also benefit from including the dynamic information as an input, seeing a $9 \%$ performance boost in the hard category for both metrics. We hypothesize that this is caused by an improved ability to detect partially occluded and far-away obstacles. In other words, if an obstacle does not have many lidar hits but we have information that it is moving, the detection can still happen. On the other hand, the detection performance for the car and cyclist classes did not benefit in general from the inclusion of the dynamic features in the dataset. This might be due to several reasons: a) these classes typically have more Lidar hits than the pedestrian class, and the extra information in the shape of occupancy data might constitute noise for the detector; b) noise in the dynamic data present towards the edges of the grid; and c) the object augmentation procedure in which the trail behind fastmoving obstacles was not artificially inserted during training.

Following the conclusions of this paper, in following work we aim to study the effect of leveraging DOGMa features for the 3D obstacle detection task in sparser point clouds, such as those obtained from Lidars with 32 or 16 layers.

Additionally, the evaluation in this paper has only been performed on the validation split of the KITTI dataset, as the test set does not provide the mapping to the raw KITTI dataset. In future work, we will perform the analysis also on the nuScenes dataset [19], which provides the preceding point clouds leading to each detection sample.

\section{ACKNOWLEDGMENT}

We would like to thank Gabriel Othmezouri from TME for the helpful discussions during the preparation of this work. Experiments presented in this paper were carried out using the Grid' 5000 testbed, supported by a scientific interest group hosted by Inria and including CNRS, RENATER and several Universities as well as other organizations (see https://www.grid5000.fr).

\section{REFERENCES}

[1] S. Ren, K. He, R. Girshick, and J. Sun, "Faster r-cnn: Towards realtime object detection with region proposal networks," in Advances in Neural Information Processing Systems 28, 2015, pp. 91-99.

[2] W. Liu, D. Anguelov, D. Erhan, C. Szegedy, S. E. Reed, C. Fu, and A. C. Berg, "SSD: single shot multibox detector," CoRR, vol. abs/1512.02325, 2015.

[3] J. Redmon, S. Divvala, R. Girshick, and A. Farhadi, "You only look once: Unified, real-time object detection," in 2016 IEEE Conference on Computer Vision and Pattern Recognition (CVPR), 2016, pp. 779788.

[4] A. Mousavian, D. Anguelov, J. Flynn, and J. Kosecka, "3d bounding box estimation using deep learning and geometry," CoRR, vol. abs/1612.00496, 2016.

[5] A. Geiger, P. Lenz, and R. Urtasun, "Are we ready for autonomous driving? the kitti vision benchmark suite," in Conference on Computer Vision and Pattern Recognition (CVPR), 2012.

[6] B. Li, "3d fully convolutional network for vehicle detection in point cloud," in 2017 IEEE/RSJ International Conference on Intelligent Robots and Systems (IROS), 2017, pp. 1513-1518.

[7] X. Chen, H. Ma, J. Wan, B. Li, and T. Xia, "Multi-view 3d object detection network for autonomous driving," in 2017 IEEE Conference on Computer Vision and Pattern Recognition (CVPR), 2017, pp. 65266534.

[8] J. Ku, M. Mozifian, J. Lee, A. Harakeh, and S. L. Waslander, "Joint $3 \mathrm{~d}$ proposal generation and object detection from view aggregation," in 2018 IEEE/RSJ International Conference on Intelligent Robots and Systems (IROS), 2018, pp. 1-8.

[9] C. R. Qi, H. Su, K. Mo, and L. J. Guibas, "Pointnet: Deep learning on point sets for $3 \mathrm{~d}$ classification and segmentation," CoRR, vol. abs/1612.00593, 2016.

[10] Y. Zhou and O. Tuzel, "Voxelnet: End-to-end learning for point cloud based 3d object detection," in 2018 IEEE/CVF Conference on Computer Vision and Pattern Recognition, 2018, pp. 4490-4499.

[11] A. H. Lang, S. Vora, H. Caesar, L. Zhou, J. Yang, and O. Beijbom, "Pointpillars: Fast encoders for object detection from point clouds," in 2019 IEEE/CVF Conference on Computer Vision and Pattern Recognition (CVPR), 2019, pp. 12689-12697.

[12] W. Luo, B. Yang, and R. Urtasun, "Fast and furious: Real time endto-end $3 \mathrm{~d}$ detection, tracking and motion forecasting with a single convolutional net," in 2018 IEEE/CVF Conference on Computer Vision and Pattern Recognition, 2018, pp. 3569-3577.

[13] S. Hoermann, P. Henzler, M. Bach, and K. Dietmayer, "Object detection on dynamic occupancy grid maps using deep learning and automatic label generation," in 2018 IEEE Intelligent Vehicles Symposium (IV), 2018, pp. 826-833.

[14] A. Petrovskaya, M. Perrollaz, L. Oliveira, L. Spinello, R. Triebel, A. Makris, J.-D. Yoder, C. Laugier, U. Nunes, and P. Bessiere, Awareness of Road Scene Participants for Autonomous Driving. London: Springer London, 2012, pp. 1383-1432.

[15] C. Coué, C. Pradalier, C. Laugier, T. Fraichard, and P. Bessière, "Bayesian occupancy filtering for multitarget tracking: An automotive application," The International Journal of Robotics Research, vol. 25, no. 1, pp. 19-30, 2006.

[16] L. Rummelhard, A. Nègre, and C. Laugier, "Conditional monte carlo dense occupancy tracker," in 2015 IEEE 18th International Conference on Intelligent Transportation Systems, 2015, pp. 2485-2490.

[17] X. Chen, K. Kundu, Y. Zhu, A. G. Berneshawi, H. Ma, S. Fidler, and R. Urtasun, "3d object proposals for accurate object class detection," in Advances in Neural Information Processing Systems 28, 2015, pp. 424-432.

[18] Y. Yan, Y. Mao, and B. Li, "Second: Sparsely embedded convolutional detection," Sensors, vol. 18, 2018.

[19] H. Caesar, V. Bankiti, A. H. Lang, S. Vora, V. E. Liong, Q. Xu, A. Krishnan, Y. Pan, G. Baldan, and O. Beijbom, "nuscenes: A multimodal dataset for autonomous driving," arXiv preprint arXiv:1903.11027, 2019. 